\title{
Obtenção e caracterização de tecido multicamadas tridirecional de fibra de aramida visando aplicação em blindagem balística
}

\author{
Obtaining and characterizing tridirectional \\ aramid fiber multilayered fabric for ballistic \\ shielding application
}

Carlos Alberto Fernandes Marlet ${ }^{1}$, Mirabel Cerqueira Rezende ${ }^{1}$,

\author{
${ }^{1}$ Instituto de Ciência e Tecnologia/Universidade Federal de São Paulo - ICT/UNIFESP, Rua Talim, 330, CEP:^12231-280, \\ São José dos Campos, São Paulo, Brasil. \\ e-mail: carlosmarlet0@gmail.com, carlos.marlet@unifesp.com, mirabelcr@gmail.com
}

\begin{abstract}
RESUMO
O uso de tecidos no processamento de materiais compósitos já é uma realidade, em função das atraentes propriedades mecânicas que esse tipo de reforço confere aos compósitos laminados e à maior facilidade de processamento. No entanto, os compósitos laminados são suscetíveis a falhas por delaminação entre as camadas. Nesse sentido, a utilização de reforços baseados em tecidos multicamadas tridirecionais (tecidos 3D), com filamentos em direções ortogonais, pode contribuir para um melhor desempenho do compósito, com a redução deste tipo de falha. Porém, o acesso a reforços 3D ainda é muito restrito ao nível mundial, devido à complexidade tecnológica envolvida no seu processamento. Nesse escopo, o presente trabalho visa contribuir com a área de processamento de compósitos com a obtenção de um tecido 3D de fibras de aramida, obtido por meio da produção e combinação de 6 camadas de tecido bidirecional, simultaneamente à amarração das referidas camadas por filamentos do urdume, processo esse realizado em uma única etapa. Para isto, um tear convencional foi adaptado e o tecido 3D produzido foi caracterizado por meio de ensaios típicos da área têxtil, como gramatura, densidade linear e espessura, análises por estereoscopia e resistências à tração e ao impacto por queda de dardo. Análises por estereoscopia revelaram detalhes das direções transversal e ortogonal do tecido, que juntamente com os ensaios da área têxtil comprovaram o sucesso da obtenção do tecido 3D, com características físicas condizentes com o arranjo pretendido. Comparativamente, os resultados de resistência à tração mostraram que o tecido 3D possui maior elongação que o tecido 2D comercial e os ensaios de impacto com $40 \mathrm{~J}$ mostraram que o tecido 3D resistiu sem perfurar e sem a delaminação das camadas, como vantagens em relação ao tecido 2D.
\end{abstract}

Palavras-chave: Reforço tridirecional. Tecido 3D. Fibras de aramida. Tecido multicamadas tridirecional.

\begin{abstract}
The use of fabrics in the processing of composite materials is already a reality due to the attractive mechanical properties that this type of reinforcement adds to the final material and the greater ease of processing. However, laminated composites are susceptible to failure by delamination between the layers. In this case, the use of reinforcements based on tridirectional multilayer fabrics (3D-fabrics) with filaments in orthogonal directions could better contribute in order to enhance composite performance, reducing this type of failure. However, access to $3 \mathrm{D}$-fabrics is still very restricted worldwide, due to the technological complexity involved in processing. For this purpose, the present work aims to contribute to composite processing area by producing a 3D-fabric with aramid fibers. The fabric was obtained through the production and combination of 6 layers of bidirectional fabric, simultaneously with the bonding of the layers by warp filaments, a process carried out in a single step. For this, a conventional loom was adapted and the $3 \mathrm{D}$-fabric manufactured was characterized by typical tests in the textile industry, as grammage, linear density, and thickness, additionally stereoscopy analysis, tensile and drop tests were assessed. The stereoscopic analysis has revealed details from transversal and orthogonal directions of the fabric, which associated with the textile industry test results proved the success of manufactured 3D-fabric, with physical characteristics consistent with the intended arrangement. Comparatively, the results of tensile strength
\end{abstract}


showed that the 3D-fabric has greater elongation than the commercial 2D-fabric. Under $40 \mathrm{~J}$ impact, 3D-fabric withstood without perforation and delamination between layers, an improvement when compared to $2 \mathrm{D}$-fabric. Keywords: Tridirection reinforcement. 3D-fabric. Aramid fibers. Tridirection multilayer fabric.

\section{INTRODUÇÃO}

Materiais compósitos, ou simplesmente compósitos, são definidos como materiais de engenharia, de ocorrência natural ou processados pelo homem, resultantes da combinação de duas ou mais fases constituintes, com propriedades físicas e/ou químicas diferentes e que permanecem distintos na estrutura final do material pela presença de uma interface [1]. Os materiais componentes do compósito são categorizados em dois tipos, um denominado de reforço e outro de matriz. O reforço é responsável principalmente pelo comportamento mecânico do compósito e pode ser constituído por partículas, fibras, filamentos ou tecidos. Já a matriz tem a função de transmitir ao reforço as cargas impostas ao componente, além de proteger o reforço de intempéries e abrasão [2-4]. A Fig. 1a ilustra um compósito polimérico com reforço de fibras contínuas.

Os reforços têxteis utilizados na manufatura de materiais compósitos de uso estrutural se apresentam costumeiramente na forma de filamentos contínuos e esses são classificados pela forma como estão dispostos no compósito. Denominam-se reforços laminares quando apresentam sua estrutura na forma de lâminas, com os filamentos dispostos paralelamente em uma única direção, chamados de reforços unidirecionais ou ao longo de um plano xy, sem que ocorra qualquer entrelaçamento entre eles (Fig. 1b). Os reforços filamentares contínuos podem ainda estar dispostos em diferentes direções, com os conjuntos de fios entrelaçados ortogonalmente formando uma única camada, denominados de tecido plano bidirecional ou 2D (Fig. 1c) ou, ainda, na forma de várias camadas com os filamentos entrelaçados entre elas e em várias direções, ou seja, na forma multidirecional $[3,5,6]$. A Fig. 1d ilustra um reforço multicamadas tridirecional (3D), denominado neste texto de tecido 3D [5].

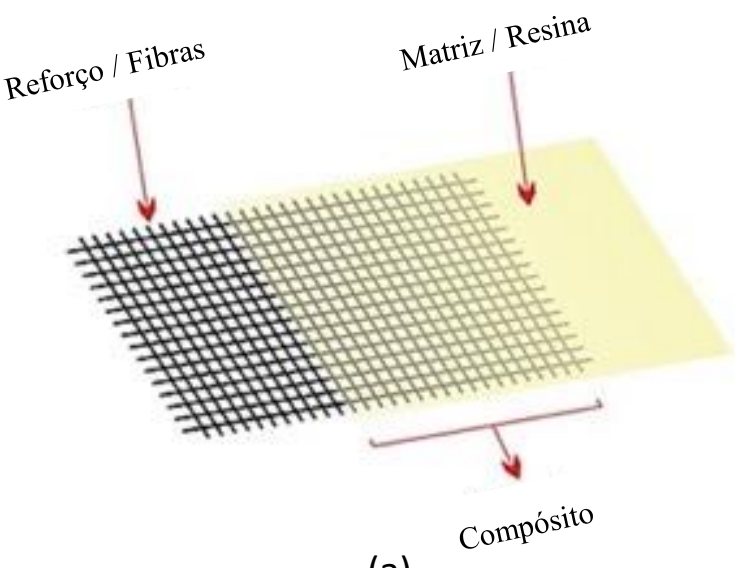

(a)

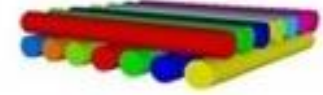

(b)

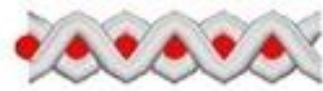

(c)

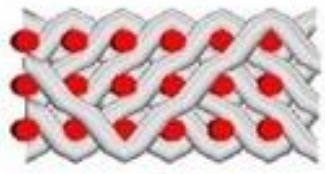

(d)

Figura 1: Esquema de um material compósito [2] (a). Tipos de reforços em materiais compósitos: (b) laminar, (c) tecido 2D e (d) tecido 3D. Baseado em [3, 5, 6].

Para algumas aplicações de engenharia que utilizam materiais compósitos, onde as peças e componentes estão sujeitos a esforços de maior intensidade e também para evitar a ocorrência de defeitos provocados pelo deslocamento das camadas durante a manufatura do componente ou durante a sua utilização, pode-se promover algum tipo de amarração das camadas de reforço [7]. O uso desse tipo de reforço favorece a obtenção de estruturas com propriedade mecânica de resistência ao cisalhamento aumentada, maior fração volumétrica do reforço, além de eliminar a condição de falha por delaminação, ponto vulnerável em se tratando de compósitos obtidos por laminação de camadas [8, 9]. Outra forma de fixação ou de amarração das camadas de tecidos para a fabricação de compósitos é por meio do processo de pinagem, onde são inseridos pinos no sentido da espessura do tecido. Porém, como avaliado por CHANG, 2006 [10], a pinagem provoca ondulações dos filamentos no plano do teci- 
do e o dobramento no sentido da espessura, além de vazios, que durante a etapa de impregnação do compósito resulta na formação de bolsas de resina.

A delaminação é uma falha que ocorre quando uma trinca, ou defeito pré-existente, se forma e se propaga através da matriz polimérica, entre as camadas de reforço. Esse tipo de defeito ocorre quando o componente em compósito sofre algum tipo de esforço elevado no sentido transversal ao reforço. A ocorrência da delaminação, causada pela ausência de fibras orientadas na direção da espessura do laminado, associada ao fato de que as camadas serem unidas apenas pela matriz polimérica, provoca perda da resistência mecânica da peça, podendo causar sua falha em serviço, especialmente por compressão no plano (flambagem) [11-14]. A manufatura de tecidos 3D, produzidos com amarração das camadas pelos próprios fios da urdidura (fio no comprimento do tecido), evitam ou reduzem a ocorrência da delaminação e, assim, propiciam a utilização de peças produzidas em material compósito em aplicações mais críticas, com melhor desempenho, eficiência e aumento da vida útil. Neste caso, pode-se citar o processamento de freios para veículos pesados de alta velocidade e blindagens balísticas [15].

\subsection{Reforços 2D e 3D}

Os reforços 2D são formados por dois conjuntos de fios ortogonais, um chamado de urdidura, posicionado no comprimento do tecido (direção $0^{\circ}$ ) e outro chamado de trama, posicionado na direção transversal (direção $90^{\circ}$ ), ou seja, na largura do tecido. A forma como os fios da urdidura e da trama se entrelaçam definem o tipo de tecido, bem como suas propriedades mecânicas. Existem inúmeras possibilidades de entrelaçamento entre os fios da urdidura e da trama, mas a mais simples e mais utilizada é chamada de tela ou plain weave (Fig. 2a). Alterandose a forma de entrelaçamento dos fios são obtidos os tecidos chamados de sarja ou twill (Fig. 2b) e o tecido tipo cetim ou satin (Fig. 2c) [7]. Outros tipos de tecidos podem ser obtidos alterando-se a forma de entrelaçamento dos fios [16-18].

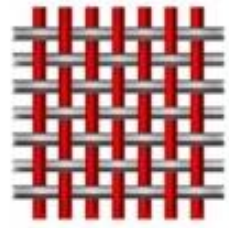

(a)

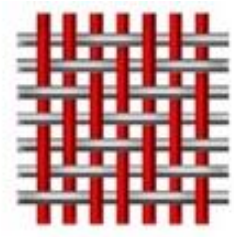

(b)

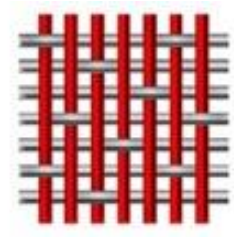

(c)

Figura 2: Tipos de tecidos 2D: (a) tela, (b) sarja e (c) cetim. Baseado em [7].

Os reforços 3D são estruturas formadas por três ou mais conjuntos de fios dispostos em direções ortogonais diferentes. Esse tipo de reforço é produzido utilizando-se dois conjuntos de fios dispostos nas direções da urdidura e trama (tecido 2D), somado a um terceiro (ou mais) conjunto de fios disposto de forma perpendicular ou em direção inclinada. Esse terceiro conjunto de fios recebe o nome de fios de amarração ou de costura. A fixação das camadas pelo uso do terceiro conjunto de fios busca aumentar a resistência aos esforços transversais sofridos pela peça, uma vez que a quantidade de fios que fixa as camadas é muitas vezes superior, tornando-a uma estrutura totalmente entrelaçada que poderia ser considerada laminar com várias camadas internas. Alterando-se a forma de entrelaçamento dos fios é possível se obter uma grande variedade de reforços 3D, como ilustra a Fig. 3 $[11,19,20]$.

$\mathrm{O}$ processo de manufatura do tecido $3 \mathrm{D}$ possui as mesmas etapas de produção do tecido $2 \mathrm{D}$, combinado com o arranjo de filamentos na terceira direção, o que torna esse processo de difícil acesso, em função da complexidade das máquinas de tecelagem necessárias para esse fim e da necessidade de conhecimentos relativos às características das fibras a serem utilizadas na produção do reforço [21]. Assim, apesar das vantagens de se utilizar reforços 3D em muitas aplicações da engenharia, a sua utilização ainda é bastante limitada, em função da dificuldade inerente do seu processo de produção $[16,22]$. Somado a isto, a revisão da literatura consultada neste estudo mostrou escassez de trabalhos técnico-científicos sobre o processamento dessa classe de material, ficando restrita a poucas informações técnicas deste tipo de reforço, cedidas por algumas empresas detentoras dessa tecnologia $[23,24]$. 
Diante do desafio existente na área de produção de reforços 3D e da limitada literatura disponível envolvendo este assunto, este trabalho de pesquisa foi motivado de ser realizado, tendo como objetivo a obtenção e a caracterização de tecidos 3D de fibras de aramida, com potencial uso na área de blindagem balística. Para isto, foi adaptada uma máquina de tecelagem específica para a obtenção do tecido 3D estudado.
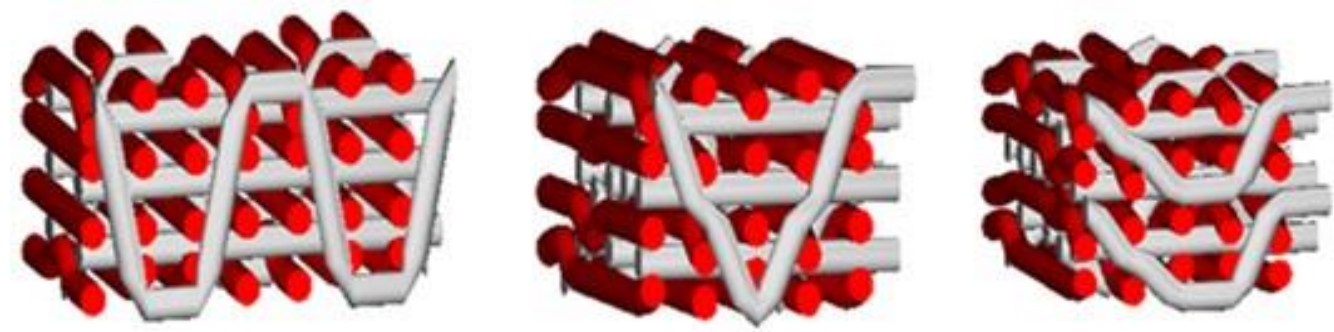

Figura 3: Exemplos de reforços 3D. Baseado em [11].

\section{MATERIAIS E MÉTODOS}

\subsection{Materiais}

Para o desenvolvimento do tecido multicamadas 3D foram utilizadas rolos de fibra de aramida com 1000 filamentos de 1570 denier por cabo e 100 torções por metro, código Kevlar K29 ${ }^{\circledR}$, da empresa DuPont do Brasil Ltda. Para efeito de comparação foi também utilizado neste estudo um tecido 2D monocamada, código 2D XPS103, da empresa DuPont do Brasil Ltda, disponível comercialmente [25]. Este tecido é baseado na mesma fibra de aramida utilizada na obtenção do tecido 3D em estudo.

\subsection{Metodologia}

\subsubsection{Obtenção do tecido 3D}

O equipamento utilizado para o desenvolvimento e obtenção do tecido multicamadas 3D estudado neste trabalho foi baseado em um desenvolvimento anterior [26], protegido via pedido de proteção intelectual MU 9000672-0 U2, 2010 [27]. O tear desenvolvido neste estudo fez uso da estrutura básica de um tear da marca Itamasa Comercial, conhecido como MAV, modelo RN 185 SL, produzido por Itapecerica Máquinas S/A. Esse sistema foi adaptado de modo a produzir tecidos multicamadas 3D. A Fig. 4 apresenta o esquema do tear modificado neste estudo. As principais modificações envolveram a substituição do rolo de urdume por cantres (ou gaiolas), formado por uma estrutura metálica com tubos e barras de aço, com capacidade para acomodar 3000 bobinas do fio da urdidura; a instalação de um pente traseiro; a substituição dos quadros de liços tradicionais por um quadro de liços desenvolvido especialmente para a produção de tecidos multicamadas 3D e o desenvolvimento de um novo sistema de inserção da trama. Com esse sistema foi possível produzir tecido com seis camadas totalmente interligadas entre si pelos próprios fios da urdidura. A Fig. 5 mostra um esquema do padrão de entrelaçamento do tecido $3 \mathrm{D}$ projetado de ser produzido neste estudo, onde os fios da urdidura promovem a amarração entre as camadas do tecido 2D simultaneamente à obtenção das seis camadas, baseado em [7].

\subsubsection{Caracterização}

A Tabela 1 mostra os ensaios realizados neste trabalho e as respectivas normas utilizadas para a caracterização do tecido 3D desenvolvido. A partir do tecido desenvolvido foram produzidos dois conjuntos de corpos de prova (CDPs) contendo 5 amostras em cada um. Um conjunto considerando a orientação dos fios da urdidura $\left(0^{\circ}\right)$ e outro considerando a orientação dos fios da trama $\left(90^{\circ}\right)$. 


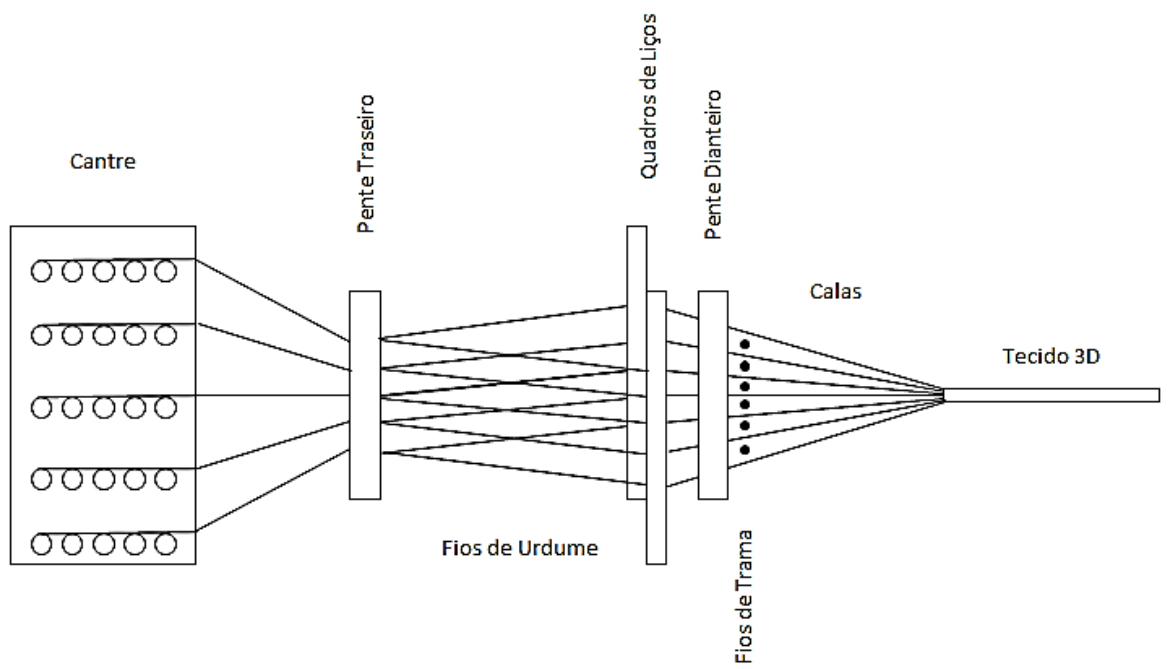

Figura 4: Esquema do tear desenvolvido e utilizado para a produção do tecido 3D neste estudo.

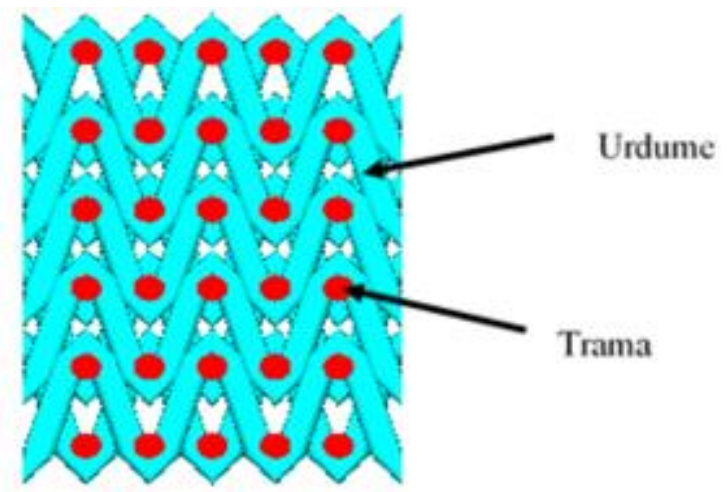

Figura 5: Esquema do entrelaçamento do tecido 3D deste estudo. Baseado em [7].

Tabela 1: Ensaios realizados para a caracterização do tecido 3D produzido.

\begin{tabular}{lcc}
\hline \multicolumn{1}{c}{ ENSAIO } & NÚMERO DE CDPS & NORMA \\
\hline Gramatura de tecido $\left(\mathbf{g} / \mathbf{m}^{\mathbf{2}}\right)$ & 5 & NBR 10591 [28] \\
Espessura do tecido $(\mathbf{m m})$ & 5 & - \\
Título dos fios $($ denier $)$ & $5^{*}$ & ASTM D1059 [29] \\
Densidade de fios do urdume e trama (fios/cm) & $5^{*}$ & ISO 7211-2 [30] \\
Densidade linear $($ crimp) dos fios no tecido $(\%)$ & $5^{*}$ & ISO 7211-3 [31] \\
Estereoscopia & $5^{*}$ & - \\
Resistência à tração & $5^{*}$ & ISO 13934-1 [32] \\
Resistência ao impacto $($ Drop test $)$ & $5^{*}$ & ASTM D7136 [33] \\
\hline
\end{tabular}

*5 CDPs para cada orientação $0^{\circ}$ e $90^{\circ}$, totalizando 10 CDPs.

\subsubsection{Gramatura}

Para os ensaios de gramatura, os CDPs dos tecidos 3D e 2D foram cortados nas dimensões de $10 \mathrm{~cm}$ x $10 \mathrm{~cm}$, perfazendo a área de $1 \mathrm{dm}^{2}$, conforme descrito na norma NBR 10591 [28]. Em seguida, foi efetuada a pesagem individual de cada amostra em uma balança Shimadzu, modelo AUW220, com precisão de $0,1 \mathrm{mg}$. A partir dos valores de massa determinados e da Equação 1, foram calculadas as gramaturas individuais das amostras, a média e o desvio padrão. 


$$
\operatorname{Gramatura}\left(g / m^{2}\right)=\frac{\operatorname{Massa}(g)}{0,01\left(m^{2}\right)}
$$

\subsubsection{Espessura}

Para a medição das espessuras dos tecidos 3D e 2D foi utilizado um micrômetro Mitutoyo. Essas medidas foram realizadas distantes $5 \mathrm{~cm}$ das bordas, de modo a evitar qualquer influência destas nas análises.

\subsubsection{Título dos fios}

$\mathrm{O}$ título dos fios correlaciona a massa pelo comprimento do filamento. $\mathrm{O}$ título pode ser expresso em denier, que representa a massa de $9.000 \mathrm{~m}$ de fio em gramas. Apesar desta informação ter sido fornecida pelo fabricante do material, estes ensaios foram realizados para a confirmação deste dado. Para isto, foram retirados dos tecidos 15 fios da trama e 15 fios do urdume para pesagem, com comprimento de $25 \mathrm{~cm}$ cada um, de acordo com a norma ASTM D1059 [29]. Em seguida, os fios foram pesados em uma balança analítica Shimadzu, modelo AUW220, com precisão de 0,1 mg. O título na unidade denier de cada amostra foi calculado de acordo com a Equação 2.

$$
\text { denier }=\frac{\text { Massa }(g) \times 9000}{\text { comprimento }(m)}
$$

\subsubsection{Densidade dos fios da trama e urdume}

O ensaio foi realizado de acordo com a ISO 7211-2 [30], com o auxílio de uma lente conta fios, instrumento padrão utilizado na indústria têxtil, para contagem de fios em um intervalo de $1,0 \mathrm{~cm}$, tanto no sentido do urdume como no da trama. A contagem foi realizada em 5 regiões diferentes dos tecidos 3D e 2D.

\subsubsection{Densidade linear (crimp) dos filamentos da trama e urdume}

A densidade linear dos filamentos da trama e do urdume considera as ondulações, também chamados de crimps, que o fio adquire no processo de tecimento. São as ondulações que o filamento adquire quando passa por baixo de um fio e por cima do fio adjacente [34], conforme ilustram as Figuras 2 e 5. Assim, este ensaio determina o aumento do comprimento total do filamento utilizado para gerar as ondulações no tecido, e é dado geralmente em porcentagem. Para este ensaio foram utilizadas amostras de tecido de $250 \mathrm{~mm}$ de comprimento por $25 \mathrm{~cm}$ de largura, conforme especificado pela norma ISO 7211-3 [31]. Para esta determinação foram extraídos filamentos na direção do comprimento da amostra, submetendo-os sob a ação de um peso de $10 \mathrm{~g}$, de modo que ficassem completamente estirados. A média calculada é obtida em relação ao comprimento da amostra.

\subsubsection{Resistência à tração}

Os ensaios de resistência à tração foram realizados em conformidade com a norma ISO 13934-1:2013 [32], em um equipamento de ensaio universal MTS Criterion, modelo C45.504 com célula de carga de $50 \mathrm{kN}$, modelo LPS.504, com abertura de garras $160 \mathrm{~mm}$, pré-tensão inicial de $10 \mathrm{~N}$ e velocidade de ensaio de $50 \mathrm{~mm} / \mathrm{min}$. Para este ensaio foram utilizados 10 CDP's de $25 \mathrm{~mm}$ x $250 \mathrm{~mm}$ para cada tecido (3D e 2D), sendo 5 na direção do urdume e 5 na direção da trama.

\subsubsection{Resistência ao impacto}

Para o ensaio de impacto (Drop test) foi utilizado um equipamento da Instron, modelo CEAST 9340, de acordo com com anorma ASTM D7136 [33]. Os ensaios foram realizados utilizando um impactador de $32 \mathrm{~kg}$, que corresponde a um impacto de $340 \mathrm{~J}$ de energia, e a velocidade de $4,67 \mathrm{~m} / \mathrm{s}$. No ensaio do tecido 2D, 6 camadas foram empilhadas, de modo a ter um número de camadas equivalentes nos ensaios. Neste caso, as bordas do tecido 2D foram impregnadas com resina epóxi, formando uma moldura, para manter as camadas fixas. A Fig. 6 mostra um CDP de tecido 3D posicionado na máquina antes e após o ensaio de impacto. 


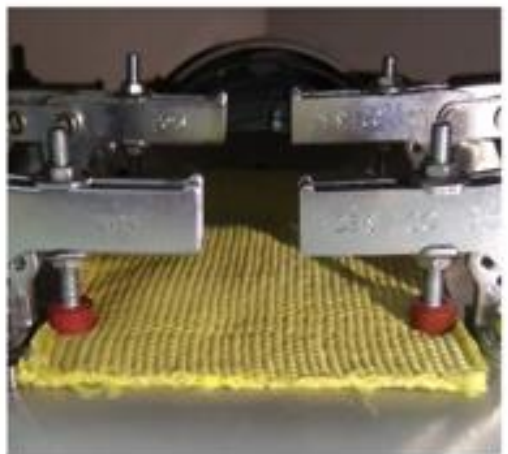

(a)

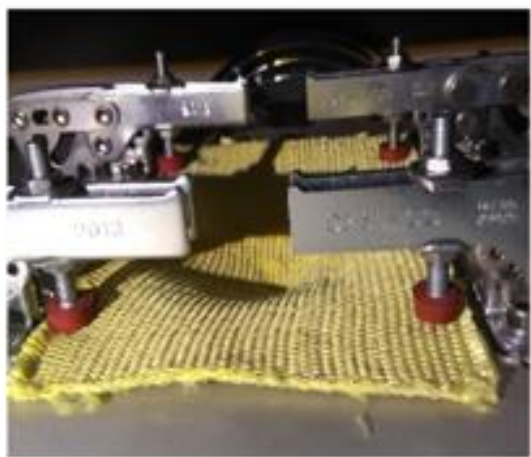

(b)

Figura 6: CDP do tecido 3D posicionado no equipamento de impacto: (a) antes e (b) após o ensaio.

\subsubsection{Estereoscopia}

As análises de estereoscopia foram realizadas utilizando-se um estereoscópio portátil, tipo caneta Instrutherm, modelo MP-150. Imagens de diferentes regiões dos tecidos 2D e 3D em estudo foram obtidas, visando mostrar o entrelaçamento dos fios da trama e do urdume dos respectivos tecidos.

\section{RESULTADOS E DISCUSSÃO}

A Fig. 7 mostra imagens representativas do tecido multicamadas 3D, cuja espessura medida foi de 3,7 $\pm 0,1 \mathrm{~mm}$, obtido a partir da tecelagem de fibras de aramida. A Fig. 7a mostra a superfície do tecido, onde se pode observar o entrelaçamento dos fios do urdume e da trama do tecido com um padrão tipo tela ou plain weave. O padrão tipo tela foi utilizado para a obtenção de cada uma das seis camadas, com distribuição homogênea dos fios da trama e do urdume. A Fig. 7b mostra uma vista da seção transversal do tecido no sentido do urdume, onde se observa a evolução dos fios do urdume ao longo do comprimento do tecido. A evolução dos filamentos neste sentido é responsável pela amarração entre as camadas do tecido. A Fig. 7c mostra a secção transversal do tecido no sentido da trama, onde se verifica a evolução do fio aproximadamente retilínea, ou seja, o fio da trama não faz a amarração entre as camadas.

$\mathrm{O}$ tecido 3D desenvolvido neste trabalho apresentou excelente manuseio e boa estabilidade dimensional. Essas características são muito favoráveis para a manufatura de peças em material compósito, quando comparada com a manufatura de componentes com tecidos convencionais $2 \mathrm{D}$, pois o tecido multicamadas $3 \mathrm{D}$ processado possui travamento mecânico, promovido pelos próprios fios da urdidura. Esse travamento das camadas evita a deformação da peça em material compósito durante as etapas de empilhamento do tecido e de infusão da resina, por exemplo, além de reduzir ou mesmo eliminar falhas por delaminação no uso final do componente processado.

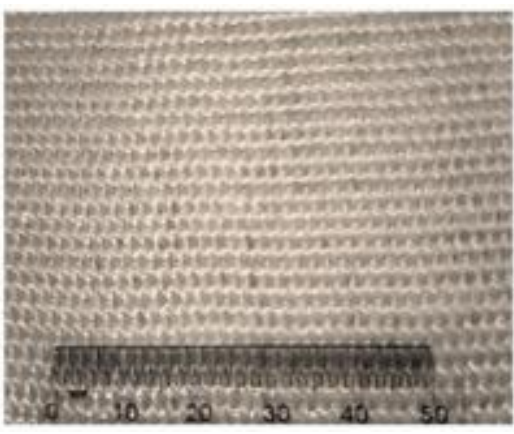

(a)

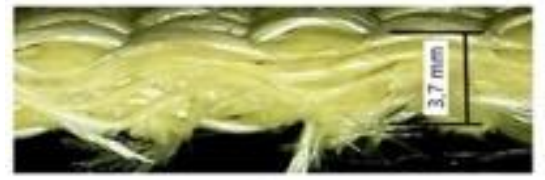

(b)

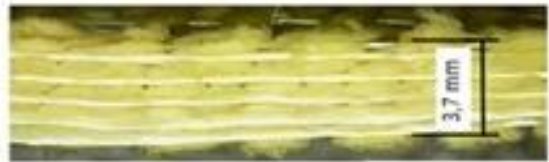

(c)

Figura 7: Vista do tecido multicamadas 3D obtido: (a) da camada superior, (b) da seção transversal no sentido do urdume e (c) no sentido da trama. 
ADANUR e SHALABY [34] citam que a costura na fabricação de compósitos acarreta no aumento da resistência à delaminação, com a vantagem do controle da densidade de pontos da costura, da orientação dos fios, com a obtenção de uma estrutura 3D formada por várias camadas de tecidos e fios. Porém, no caso da costura, os fios do tecido podem ser danificados pela penetração da agulha durante o processo de costura, reduzindo a resistência e integridade estrutural do compósito, como menciona [35, 36]. Desvantagem essa que o tecido desenvolvido neste trabalho não apresenta, pois o travamento das camadas ocorre pelos próprios fios da urdidura.

$\mathrm{O}$ processo de manufatura do tecido 3D desenvolvido neste estudo evita a necessidade de amarração das camadas por costura ou pinagem. Dessa forma, o processo produtivo se torna mais rápido, já que reduz etapas de processamento de empilhamento e amarração das camadas. Outro ponto importante a ser ressaltado é o fato que tanto o processo de costura como o de pinagem serem efetuados com espaçamentos pré-determinados e utilizam apenas alguns filamentos [10], enquanto, que a amarração com os próprios fios da urdidura se dá de maneira homogênea em toda a largura e comprimento do tecido 3D produzido. Nesse sentido, a obtenção de tecidos 3D com amarração pelo próprio fio da urdidura, como realizado neste estudo, é uma vantagem.

A Tabela 2 apresenta os resultados obtidos na caracterização física do tecido multicamadas 3D desenvolvido neste trabalho, em comparação com o tecido 2D monocamada 2D XPS103, disponível comercialmente [25], lembrando que ambos os tecidos foram produzidos com a mesma fibra de aramida.

Tabela 2: Características físicas do tecido 3D processado e de um tecido 2D comercial, ambos com a mesma fibra de aramida.

\begin{tabular}{lcc}
\hline \multicolumn{1}{c}{ Características } & Tecido 3D & Tecido 2D comercial \\
\hline Tipo de entrelaçamento no plano & Tela & Tela \\
Número de camadas & 6 & 1 \\
Gramatura $\left(\mathrm{g} / \mathrm{m}^{2}\right)$ & $1458,5 \pm 14,8$ & $221,0 \pm 5,6$ \\
Espessura $(\mathrm{mm})$ & $3,7 \pm 0,2$ & $0,46 \pm 0,07$ \\
Título do fio da urdidura (denier) & $1575,3 \pm 18,0$ & $1565,0 \pm 20,0$ \\
Título do fio da trama (denier) & $1573,6 \pm 19,4$ & $1571,6 \pm 19,4$ \\
Densidade dos fios da urdidura por camada (fios/cm) & $8,4 \pm 0,5$ & $6,6 \pm 0,2$ \\
Densidade dos fios da trama por camada (fios/cm) & $6,2 \pm 0,4$ & $6,1 \pm 0,4$ \\
Densidade linear de fios de urdume (crimp) $(\%)$ & $7,2 \pm 2,6$ & $0,94 \pm 0,25$ \\
Densidade linear de fios de trama (crimp) $(\%)$ & $1,5 \pm 0,3$ & $1,0 \pm 0,2$ \\
\hline
\end{tabular}

Considerando que a gramatura é a relação da quantidade de material por área, quanto maior a gramatura maior é o peso do tecido. O resultado médio da gramatura do tecido multicamadas 3D $\left(1458,5 \pm 14,8 \mathrm{~g} / \mathrm{m}^{2}\right)$ apresenta-se aproximadamente 6,6 vezes maior que o obtido para uma única camada do tecido $2 \mathrm{D}\left(221,0 \mathrm{~g} / \mathrm{m}^{2}\right)$. Comparativamente, essa relação é aproximadamente a equivalência entre uma camada do tecido 3D e 6 camadas do tecido 2D, lembrando que o tecido 3D é formado por 6 camadas de tecido 2D amarradas pelo fio da urdidura. Com relação à espessura, o tecido 3D apresenta a espessura de 3,7 mm e o tecido 2D a espessura de $0,46 \mathrm{~mm}$. Comparativamente, verifica-se que o tecido 3D apresenta uma espessura maior do que à equivalente às 6 camadas do tecido 2D somente empilhadas. Esta característica se deve à forma de como os fios da urdidura são posicionados durante o processo de manufatura para obter o entrelaçamento desejado entre as camadas do tecido 3D, ou seja, a amarração das camadas contribui para o aumento relativo da espessura do tecido 3D com 6 camadas $2 \mathrm{D}$ amarradas.

Outra característica que mostra a evolução diferenciada dos fios do urdume no tecido 3D é a densidade linear (crimp). As análises da densidade linear do tecido 3D desenvolvido mostraram maior consumo de filamentos do urdume $(7,2 \%)$ contra $0,94 \%$ do tecido $2 \mathrm{D}$, diferença justificada pela evolução desenvolvida na manufatura do tecido 3D. Com relação à densidade linear dos fios da trama também se observa um maior valor para o tecido $3 \mathrm{D}(1,5 \%)$ em relação ao $2 \mathrm{D}(1,0 \%)$, diferença essa menos significativa, mas também atribuída ao arranjo dos filamentos no tecido 3D. 
Em relação aos títulos dos filamentos da urdidura e da trama para os tecidos 3D e 2D não se observam diferenças significativas (1565,0 a 1575,3 denier), considerando que o mesmo tipo de filamento de aramida foi utilizado na obtenção dos dois tipos de tecidos em questão. Os valores obtidos experimentalmente são concordantes com o fornecido pelo fabricante (1570 denier por cabo).

Com relação à densidade dos fios da trama por camada têm-se valores equivalentes $(\sim 6,2$ fios/cm) para os dois tecidos, já que no plano tem-se o mesmo arranjo tipo tela, ou seja, a evolução desses fios no tecido 3D (na camada) é equivalente à do tecido 2D. Já a densidade dos fios da urdidura por camada mostra um valor maior para o tecido $3 \mathrm{D}(8,4 \pm 0,5$ fios/cm) em relação ao tecido $2 \mathrm{D}(6,6 \pm 0,2$ fios/cm), devido ao fio da urdidura estar sendo utilizado para fazer a amarração das 6 camadas, em concordância com os dados de crimp para os dois tecidos analisados. Neste caso, a amarração do tecido 3D utiliza maior quantidade de fio da urdidura.

A Tabela 3 apresenta os valores médios dos ensaios de resistência à tração realizados nos tecidos 3D e 2D. A análise destes resultados foi realizada através dos valores de força máxima, uma vez que não há uma seção transversal sólida do corpo de prova para o cálculo da área e da tensão. O tecido 3D apresenta valores médios de força máxima média de $12.571 \pm 625 \mathrm{~N}$ e elongação de $8,1 \pm 2,4 \%$ na direção do urdume e de $10.256 \pm 422 \mathrm{~N}$ e $4,8 \pm 2,7 \%$ de elongação no da trama. Estatisticamente, esses resultados mostram que o tecido 3D apresenta melhor desempenho na direção do urdume e também maior elongação. Já o tecido 2D apresenta força máxima média de $823 \pm 197 \mathrm{~N}$ na direção do urdume e $714 \pm 103 \mathrm{~N}$ no sentido da trama e elongação de $0,9 \pm 0,2 \% \mathrm{em}$ ambas as direções. Neste caso, os valores obtidos não apresentam diferença estatística significativa para indicar melhor desempenho na direção do urdume. Comportamento esse esperado, considerando que o tecido 2D (tipo tela) é equilibrado nas duas direções. No entanto, sabe-se que na direção do urdume podem-se ter valores ligeiramente maiores em relação aos da trama, devido ao processo de manufatura do tecido trabalhar com o fio do urdume com maior tensão que o fio da trama, o que pode favorecer um maior alinhamento dos filamentos constituintes do fio, gerando maior resistência do tecido nesse sentido.

Considerando que as gramaturas dos tecidos 3D e 2D são diferentes (Tabela 2), as forças máximas obtidas para os dois tecidos foram divididas pela massa dos corpos de prova ensaiados, de modo a se fazer a comparação das resistências específicas. A análise desses dados mostra que o tecido 3D apresenta uma resistência específica aproximadamente 2,6 vezes maior no sentido do urdume e 2,4 vezes maior no da trama, em relação ao tecido $2 \mathrm{D}$, evidenciando o melhor desempenho mecânico em tração do tecido 3D processado.

Tabela 3: Valores de resistência à tração dos tecidos $2 \mathrm{D}$ e 3D.

\begin{tabular}{lcccccc}
\hline & \multicolumn{3}{c}{ Tecido 3D } & \multicolumn{2}{c}{ Tecido 2D } \\
\hline & $\begin{array}{l}\text { Força máxima } \\
\text { na ruptura }(\mathrm{N})\end{array}$ & $\begin{array}{c}\text { Elongação } \\
(\%)\end{array}$ & $\begin{array}{c}\text { Força máxima/massa } \\
\text { do CDP }(\mathrm{N} / \mathrm{g})\end{array}$ & $\begin{array}{c}\text { Força máxima } \\
\text { na ruptura }(\mathrm{N})\end{array}$ & $\begin{array}{c}\text { Elongação } \\
(\%)\end{array}$ & $\begin{array}{c}\text { Força máxima/massa } \\
\text { do CDP (N/g) }\end{array}$ \\
\hline Trama & $10256 \pm 422$ & $4,8 \pm 2,7$ & $1236 \pm 54$ & $714 \pm 103$ & $0,9 \pm 0,2$ & $517 \pm 80$ \\
Urdume & $12571 \pm 625$ & $8,1 \pm 2,4$ & $1493 \pm 79$ & $823 \pm 197$ & $0,9 \pm 0,2$ & $580 \pm 136$ \\
\hline
\end{tabular}

A análise dos CDPs de tecido 2D após os ensaios de tração, nas direções do urdume e da trama, mostrou que esses apresentaram uma desestruturação acentuada do reforço, principalmente os CDPs extraídos na direção do urdume. Comportamento semelhante, porém, menos acentuado, também foi observado para o tecido 3D.

Com relação ao ensaio de impacto, a amostra de tecido 2D, com seis camadas empilhadas, foi perfurada com o impacto de $40 \mathrm{~J}$, após uma deformação de aproximadamente $22 \mathrm{~mm}$. Já a amostra de tecido 3D sofreu uma deformação de $15 \mathrm{~mm}$ de profundidade (Fig. 8a), porém sem a perfuração do material (Fig. 8b). Esta figura refere-se ao corte transversal de um CDP após o ensaio de impacto. A Fig. 8c apresenta em maior detalhe a seção transversal do tecido 3D após o impacto, onde não se observa a presença de delaminação. Os CDPs extraídos na direção do urdume é que apresentaram maiores danos, em relação aos cortados na direção da trama. Isto se deve ao fato do fio da trama ficar mais fixo e sem muitas ondulações, como mostra da Fig. 7c, conferindo maior rigidez ao tecido 3D nesta direção. Um resultado bastante relevante observado no ensaio de impacto é que nenhum dos corpos de prova ensaiados com o tecido 3D apresentou delaminação entre as camadas, evidenciando o efeito da amarração realizada com o fio do urdume. Este resultado é significativo quando se pretende fazer uso de reforços 3D para a produção de peças em compósitos, por exemplo, em sistemas de freios [37, 38] ou em blindagens balísticas [39, 40]. 


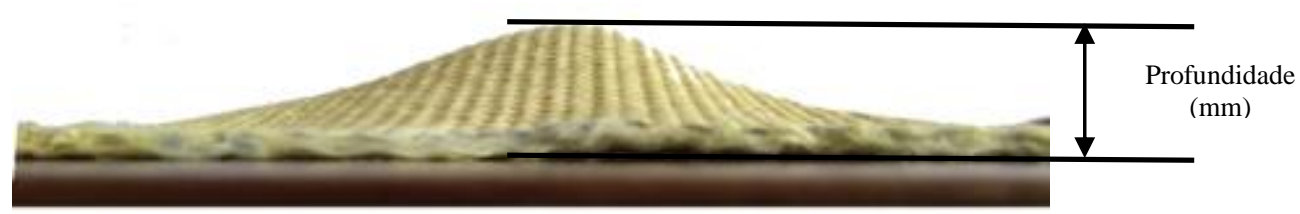

(a)

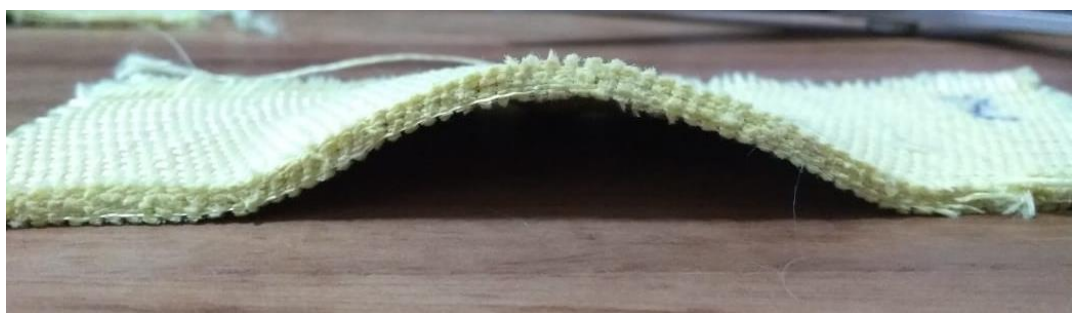

(b)

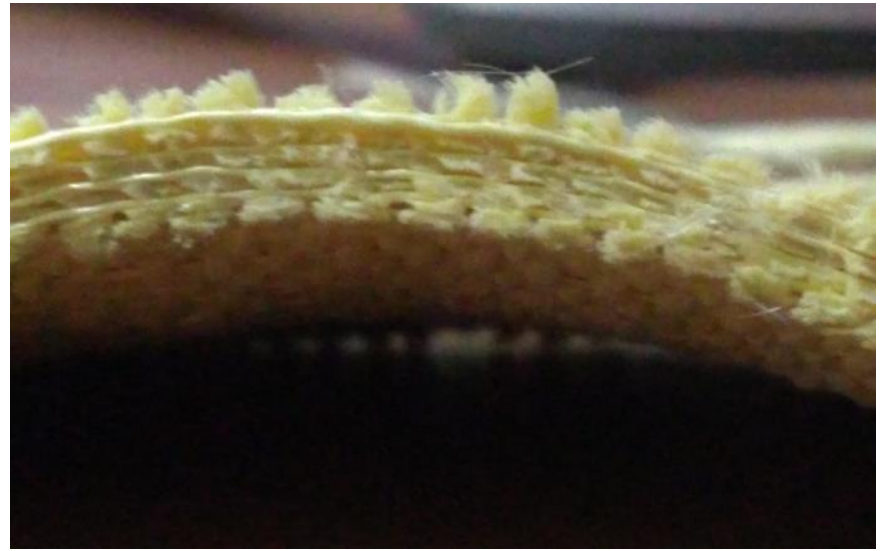

(c)

Figura 8: Vista de um CDP do tecido 3D: (a) após o ensaio de impacto, (b) corte transversal do CDP e (c) detalhe da região impactada, sem a presença de delaminação.

\section{CONCLUSÕES}

Os resultados das caracterizações física, estereoscópica e mecânica mostraram que o tecido 3D proposto foi obtido com sucesso. A gramatura média do tecido multicamadas 3D é 6,6 vezes maior que a determinada para o tecido 2D. Essa relação é aproximadamente a equivalência entre uma camada do tecido 3D e 6 camadas do tecido 2D, indicando que o tecido 3D proposto neste estudo foi obtido com sucesso. Os valores de espessura e densidade linear (crimp) do tecido 3D em relação ao 2D também evidenciam o sucesso da manufatura do tecido multicamadas proposto. As análises por estereoscopia mostraram a combinação das 6 camadas de tecido 2D, tipo tela, amarradas entre si pelos próprios fios da urdidura, como almejado no objetivo deste trabalho. Os ensaios de resistência à tração mostraram que a força máxima específica é superior na direção do urdume, em relação à da trama para o tecido 3D, com elongação significativamente maior ( 9 vezes) na direção do urdume, em relação ao tecido 2D. Comportamento esse atribuído ao arranjo do reforço utilizar maior teor de fio de urdume para fazer a amarração entre as camadas. Um resultado relevante é a ausência de delaminação e de perfuração dos corpos de prova com tecido 3D ensaiados em impacto por queda de dardo.

\section{AGRADECIMENTOS}

Os autores agradecem à FAPESP (Processos PIPE II nº. 2015/50065-7 e 2018/09531-2), CNPq (Processo 305123/2018-1) e à CAPES (001) pelos aportes financeiros concedidos, à empresa TECPLAS INDÚSTRIA E COMÉRCIO LTDA pela disponibilização de sua infraestrutura e ao Laboratório de Estruturas Leves/IPT pelos ensaios de impacto. 


\section{BIBLIOGRAFIA}

[1] HU N., Composites and their applications, ebook, InTech, Croácia, 2012.

[2] Site da empresa OXYBLACK - Composite, Ltda, disponível em https://www.oxyblack.com/index.php/pt/compositos/o-que-sao, acessado em 27/11 2019.

[3] DUARTE, L., Compósitos sustentáveis de retalhos no CONEM 2012, disponível em: https://lucianaduarte.wordpress.com/2012/01/25/compositos-sustentaveis-de-retalhos-no-conem-2012/, acessado em $26 / 11 / 2019$.

[4] COELHO, A.C.V., Unidade 19 Materiais Compósitos, material da disciplina PMT 3100 - Fundamentos de Ciência e Engenharia dos Materiais da Escola Politécnica da Universidade de São Paulo, 2017

[5] RANA, S., FANGUEIRAS, R., Fibrous and Textile Materials for Composite Applications, 1 ed., Springer, Singapura, 2016.

[6] CORBINI, A.C., KECECI, A., BOUSSUL, F., Ferreira M., Soulat D., Engineering Design and Mechanical Property Charactherisation of $3 D$ Warp Interlock Woven Fabrics, Appl Compos Mater 25,811-822, Springer Nature B. V., 2018.

[7] BILISIK, K., "Advances in modern woven fabrics technology", In: INTECH EUROPE, UK, Multiaxis three dimensional (3D) woven fabric., capítulo 5, Vassiliadis S. G., pp. 79-106, 2011.

[8] MATUSIAK, M., SIKORSKI. K,, WILK. E., Innovative woven fabrics for therapeutic clothing, In: Innovations in Textile Materials \& Protective Clothing, edited by Bartkowiak G, Frydrych I, Pawłowa M, CIOP-PIB Warsaw, pp. 89-106, 2012.

[9] VIOLEAU, D., DAGHIA, F., GENDRE, L., Les dégradations des matériaux composites: les phénomènes physiques, publicado por Universidade Paris-Saclay, disponível no site : https://eduscol.education.fr/sti/si-enscachan/ressources_pedagogiques/les-degradations-des-materiaux-composites-les-phenomenesphysiques\#description, acessado em 18/01/2016.

[10] CHANG, P., The mechanical properties and failure mechanisms of Z-pinned composites, Thesis Doctor of Philosophy, School of Aerospace, Mechanical and Manufacturing Engineering, RMIT University, Melbourne, Austrália, 2006.

[11] REIS, J.F., ABRAHAO, A.B.M., COSTA, M.L., et al., Avaliação da Resitência Interlaminar do Compósito PEI/Fibra de Carbono Soldado pelo Método de Resistência Elétrica, In: Soldagem\&Inspeção, ed. 21, n. 3, pp. 387-404, 2016.

[12] CAMPOS, V.R., Análise de Ligação Mecânica em Compósito Polimérico Reforçado com Tecido de Fibras de Sisal, dissertação de Mestrado, Universidade Federal da Bahia, 2016.

[13] MORAIS, W.A., D'ALMEIDA, J.R..., Comparação do Comportamento à Flexão com Restriçõesà Deflexão de Placas de Compósitos de Matriz Polimérica Epoxídica em Função do Tipo de Fibra de Reforço; In: Polímeros : Ciência e Tecnologia, v. 13, n. 3, pp. 181-187, 2003.

[14] DURÃO, L.M.P., GONÇALVES, D.J.S., TAVARES, J.M.R.S., et al., Delaminação na Furação de Laminados Carbono/Epoxídico; Trabalho apresentado no Congresso Iberoamericano de Ingeniería Mecánica CIBIM 10; Porto, Portugal, 2011.

[15] DE BAERE, I., JACQUES, S., VAN PAEPEGEM, W., et al., Study of the mode I and mode II interlaminar behaviour of a carbon fabricreinforced thermoplastic, In: Polymer Testing, ed. 31, n. 2, pp. 322-332, 2012.

[16] DHOOT, N.S., An Overview on Representation of Woven Fabric Structure, In: Textile Review, edição janeiro, 2013.

[17] CREPALDI, E.A., Influência do fator de cobertura nas propriedades de permabeabilidade de tecidos planos, dissertação de Mestrado, Escola de Artes Ciências e Humanidades, Universidade de São Paulo, São Paulo, SP, Brasil, 2017.

[18] UNAL, P.G., 3D Woven Fabrics, In : Woven Fabrics, Edit. InTech, Croácia, 2012.

[19] MANDOT, A., PARMAR, S., LUNAGARIA, D., et al., 3-Dimensional Weaving - Taking Textiles to the Next Dimension, In: Textile Review em www.fibre2fashion.com, acessado em 28/08/ 2019, 2012.

[20] BOUSSU, F., CRISTIAN, I., NAUMAN, S., General definition of 3D warp interlock fabric architecture, In: Elsevier journal, Composites Part B 81, pp. 171 a 188, 2015.

[21] CHEN, X., TAYLOR, L.W., TSAI, L.J., An overview on fabrication of three-dimensional woven textile preforms for composites, In: Textile Research Journal, edição 81, n. 9, pp. 932-944, 2011. 
[22] WAMBUA, P.M., ANANDJIWALA, E., A review of preforms for the composites industry, In: Journal of Industrial Textiles, v. 40, n. 4, abril de 2011.

[23] MEP-OLBO GmbH. Technical textiles. In: http://www.technicaltextilesfinder.com/germany/fulda/fabricsby-technology/mep-olbo-gmbh. Fulda/Alemanha. Acessado em 01/07/2020.

[24] 3tex Inc. In: https://www.compositesworld.com/suppliers/3tex. Rutherfordton/USA. Acessado em 01/07/2020.

[25] DuPont., DuPont Kevlar ${ }^{\circledR} X P^{\mathrm{TM}}$ S103 Product Specification. Disponível em:

http://www.dupont.com/content/dam/dupont/productsandservices/fabricsfibersandnonwovens/fibers/documents/ DSP_Kevlar_XPS103_datasheet_K23846_2.pdf, acessado em março de 2019.

[26] MARLET, C.A.F., Desenvolvimento de tecido multicamadas tridirecional (3D) para ser utilizado como reforço em materiais compósitos, dissertação de M.Sc, Instituto Tecnológico de Aeronáutica (ITA), São José dos Campos, SP, 2014.

[27] MARLET, C.A.F., Máquina de tecimento contínuo e ininterrupto para fabricação de tecidos tridimensionais multicamadas, Carlos Alberto Fernandes Marlet, BR, MU 9000672-0 U2, depositada em 17/05/2010 e concedida em 03/01/2012.

[28] ASSOCIAÇÃO BRASILEIRA DE NORMAS TÉCNICAS, Materiais têxteis - determinação da gramatura de tecido, NBR 10591, Rio de Janeiro, 1988, 2 p.

[29] ASSOCIAÇÃO BRASILEIRA DE NORMAS TÉCNICAS, Standard test method for yarn number based on short-length specimens, ASTM D1059-17, Rio de Janeiro, 2017, 6 p.

[30] INTERNATIONAL ORGANIZATION FOR STANDARDIZATION, Textiles - Woven fabrics - Construction - Methods of analysis - Part 2: Determination of number of threads per unit length, ISO 7211-2, Genebra, Suíça, 1984, 6 p.

[31] INTERNATIONAL ORGANIZATION FOR STANDARDIZATION, Textiles - Woven fabrics - Construction -Methods of analysis - Part 3: Determination of crimp of yarn in fabric, ISO 7211-3, Genebra, Suíça, 1984, $3 \mathrm{p}$.

[32] INTERNATIONAL ORGANIZATION FOR STANDARDIZATION, Textiles - Tensile properties of fabrics - Part 1: Determination of maximum force and elongation at maximum force using the strip method, ISO 13934-1, Genebra, Suíça, 1999, 12 p.

[33] ASSOCIAÇÃO BRASILEIRA DE NORMAS TÉCNICAS, Standard test method for measuring the damage resistance of a fiber-reinforced polymer matrix composite to a drop-weight impact even, ASTM D7136M12, Rio de Janeiro, 2012, 16 p.

[34] ADANUR S., SHALABY S.E., Design and Manufacture of Stitch Bonded Textile Composites, In: USEgypt Science and Technology Fund Contract n ${ }^{\circ}$ MAN2-002-98, Egito, 2002.

[35] PEERZADA, M.H., ABBASI, S.A., KHATRI, A., Effect of weave structure on tensile strength and yarn crimp of three-dimensional fibre woven fabric, In: Sci.Int.(Lahore), 24(1), pp. 47-50, Universiti Malaya, Malásia, 2012.

[36] MCCLAIN, M., GOERING, J., Rapid assembly of fiber preforms using 3D woven components, In: Sampe journal, ed. 49(6), 2012.

[37] DANTE, R.C., Handbook of friction materials and their applications, Woodhead Publishing in Materials, Elsevier, 2016.

[38] BALASUBRAMANIAN, M., "Fibrous and Textiles Materials for Composite Applications", Introduction to composite materials, capítulo 1, Universidade do Minho, Portugal, 2016.

[39] PANDEY, D.N., Desigung 3Dimensional Woven Fabric Based on Conventional Techinque for Geo Textiles, In: International Journal of Applied Engineering Research, vol. 7, nº 11, 2012.

[40] BILISIK, K., KARADUMAN, N.S., BILISIK N. E., "Fibrous and Textiles Materials for Composite Applications", Fiber Architectures for Composite Applications, capítulo 5, Universidade do Minho, Portugal, 2016.

ORCID

Carlos Alberto Fernandes Marlet

Mirabel Cerqueira Rezende https://orcid.org/0000-0003-1191-1435

https://orcid.org/0000-0002-3735-8765 